\title{
Experimental Studies of Perturbations in Ionospheric Plasma
}

\author{
L. H. Heisler ${ }^{1}$
}

(Received August 20, 1964)

\begin{abstract}
An account is given of some of the more important experimental procedures for observing perturbations in ionospheric plasma, and mention made of significant data derived from these observations.

Particular reference is made to investigations recently instigated using a new type of ionospheric recorder or ionosonde which is providing valuable data on many aspects of the phenomenon.
\end{abstract}

\section{Introduction}

The presence of perturbations in the ionospheric medium has been known for some time, however, it is not until recently since the advent of satellites and subsequent availability of signals from a convenient source embedded in the plasma that a full appreciation has been gained of the variety of sizes and number of irregularities present, and their extent throughout the ion density distribution. Titheridge [1963], using measurements of electron content between satellite and ground-based observing station, has been able to show that perturbations are present in the ionospheric medium ranging in size from 5 to $500 \mathrm{~km}$. Moreover, he found that these occurred singly, in groups of a similar size, or even in the form of small perturbations superimposed on large perturbations. Observations by the Alouette topside sounder also confirm the presence of a similar variety and number of perturbations above the height of maximum ion density [Calvert and Schmid, $1964]$.

Most of our observations of ion density irregularities however are confined to conventional groundbased observations. It is a feature of these perturbations that their spatial distribution is changing continuously; hence studies of short term temporal variations and for that matter longer diurnal and seasonal variations are somewhat limited by the transient nature of satellite observations. Furthermore, in the lower ionospheric regions, direct observation of the phenomenon of sporadic $E$, which must also be considered as a perturbation within the ion density medium, is only possible by transient rocket samplings. The continuity of data necessary for these studies can therefore only be obtained by ground based observations.

\footnotetext{
1 Radio Research Board Laboratory, School of Electrical Engineering, Uni-
} versity of Sydney, Sydney, Australia.
Conventional forms of these observations rely implicitly upon measurements of the ionosphere made by a probing radio-frequency ray radiated vertically from the earth's surface. The plasma is usually considered as being horizontally stratified or consisting of a series of surfaces of equal ion density (isoionic surfaces) parallel to the surface of the ground. Reflection occurs when the ray falls normally upon a surface of plasma frequency equal to the radiated frequency, at the zenithal point above the recording station. However, the presence of ion density perturbations in the medium modify the effective reflecting surfaces introducing "ripples" and curvature which result in diffraction, focusing, and nonvertical reflection processes, readily manifested as irregular features on related records.

It is the purpose of this paper to discuss some of the experimental techniques used to study perturbations in ionospheric plasma, summarizing some of the more important results, in particular those obtained from a new type of ionosonde, which are proving invaluable in elucidating many problems associated with the phenomena.

\section{Single Frequency Observations of Iono- spheric Perturbations}

\subsection{Variations in Virtual Height}

Munro [1958] has made extensive studies of perturbations by means of observations at spaced stations (40 km separation) on a single frequency of $5.8 \mathrm{Mc} / \mathrm{s}$. He observed variations in curvature of effective reflecting surfaces as complexities on continuous records of virtual height, moreover, he showed that records at each station always displayed time differences between similar phases of the same complexity indicating that observed curvatures had an apparent horizontal component of progression through the ionosphere [Munro, 1950, 1953]. From observed 
time differences and known configuration of observation points it was therefore possible to attribute a definite speed and direction to the motion assuming of course that induced distortion was frontal in form and direction of movement was perpendicular to this front. All observations made by Munro refer to the $F$ region and were made mainly during daylight hours as occurrence of complexities at night is infrequent, moreover, since manifestation of the phenomenon on records as a complexity is largely a function of spatial geometry of isoionic surfaces. Since this in turn relies on the ion density gradient in which the perturbation appears [Munro, 1953]; absence of recorded complexities does not necessarily imply absence of perturbations in the observed part of the medium.

As in common with most other methods of observing ionospheric perturbations, the technique is sensitive only to certain size perturbations. Parameters of large perturbations cannot be determined because height changes are too complex to permit satisfactory analysis, while small perturbations are not apparent because induced height changes cannot be resolved by the recording equipment. It is usually considered that most perturbations observed by this technique are of the order $100 \mathrm{~km}$ in extent.

In measurements made at Sydney $\left(33^{\circ} 52^{\prime} \mathrm{S}\right.$, $151^{\circ} 11^{\prime} \mathrm{E}$ ) over a period of eight years, Munro [1958] found marked seasonal variations in direction of movement of perturbations observed during daylight hours. This is directed towards a mean direction $30^{\circ}$ East of north during winter, but changes abruptly during September or October to a mean direction $120^{\circ}$ East of north in summer. The change during equinoxial months of February to May is more gradual. At all times, except during equinoxial periods, there is a strong north-south component of movement. The mean speed of travel from all observations is of the order $130 \mathrm{~m} / \mathrm{s}$. As already mentioned, night observations are few, but these indicate a change in east-west component in all seasons, movement being directed towards the northwest in winter and southwest in summer. There is not much information on speeds and direction of movement from other geographical localities as this method has not been widely used by other observers. However, Price [1955] in a series of observations made at Perth $\left(31^{\circ} 57^{\prime} \mathrm{S}, 175^{\circ} 52^{\prime} \mathrm{E}\right)$ using the same technique found directions, speeds, and seasonal variations consistent with those observed by Munro. Toman [1955] made similar observations during the northern hemisphere winter in New England, U.S.A. Speeds observed ranged from 70 to $160 \mathrm{~m} / \mathrm{s}$ with a preferred direction of movement $117^{\circ}$ east of north.

One feature of these observations is that a time difference is always observed between appearance of the complexity on the ordinary and extraordinary ray trace [Munro and Heisler, 1956b] even when the perturbation has an apparent movement at right angles to the magnetic meridian plane. At this latitude the magneto-ionic component reflection points are separated both horizontally and vertically in this plane, and therefore a vertical component of progression, or tilt in the wave front of the perturbation in its direction of movement, must also be present.

During observation of $F$ region perturbations using this particular method, time differences were observed at individual stations between appearance and demise of sporadic $E$ reflections. This has been used by Harvey [1955] to establish speeds and direction of apparent movement of $E_{\mathrm{s}}$ patches. Harvey found directions of movement consistently towards the northwest in all seasons, with speeds between 20 and $80 \mathrm{~m} / \mathrm{s}$.

\subsection{Rapid Fading of Radio Signals}

Because of its experimental simplicity many observers throughout the world use a method of observing perturbations originally due to Mitra [1949] sometimes with additional experimental refinements developed by Phillips [1955]. This technique relies upon observation of intensity of reflected pulse signals from the ionosphere at spaced receiving stations. It is found that variation or fading is often similar at individual sites, but with a phase displacement in time. The observed phenomenon is considered to be due to a diffraction process, resulting from the apparent movement of small perturbations within the ionospheric medium so that as previously it is possible to determine a speed and direction of movement for contributing irregularities. Spacing of observing sites of the order one wavelength of the observing frequency or less is found necessary to obtain satisfactory correlation of events.

As in the virtual height method described above this particular technique is sensitive only to certain size perturbations. Very small and very large irregularities introduce fading rates in records which are either too fast or too slow to permit satisfactory analysis. Ratcliffe [1956] has shown that perturbations observed are greater than 300 meters and possibly of the order some hundreds of meters in extent. In addition estimated parameters apply only to those perturbations which provide consistent fading patterns over the observing area. There are many cases where fading is random probably due to change in form of the irregularity as movement occurs. These cases are usually rejected and derived data is therefore somewhat selective in form. Briggs and Spencer [1954] and Shimizaki [1959] have also pointed out that there is an ever present doubt in $F$ region measurements as to whether the diffracting layer is in this region or in lower portions of the medium through which the observing signal passes. This ambiguity does not occur in the method described in section 2.1 above as observed time differences between appearance of observed complexities on ordinary and extraordinary ray traces are consistent with expected separation of reflection points in the $F$ region.

Despite these limitations, this method was almost universally used during the IGY. Unfortunately, due to the practice of most workers to deliberately 
choose low values of observing frequency so as to obtain observations of $E$ region during the daytime and $F$ region at night, there is not an extensive amount of daytime $F$ region data available. There is also considerable doubt as to the validity of the practice of combining daytime $E$ observations with nighttime $E_{s}$ observations to obtain diurnal information on movements in $E$ region. Observed movement could be occurring at different heights or even be due to entirely different processes. $F$ region observations made by this method during the TGY have been summarized by Briggs [1960]. Contrary to results derived from the technique due to Munro (2.1 above) a strong east-west component of the order $100 \mathrm{~m} / \mathrm{s}$ is always dominant, and at night this is directed towards the west in moderate and high latitudes and towards the east near the equator, a reversal taking place in the vicinity of $30^{\circ}$ magnetic latitude. A general reversal of this component also occurs during daylight hours. A small north-south component of the order 20 to $40 \mathrm{~m} / \mathrm{s}$ is present at night at high latitudes directed towards the equator, while during daylight hours this component velocity is very small.

Observations of $E$ region during the TGY have been summarized by Shimazaki [1960]. During winter a strong southerly component is present at all latitudes, and the east-west component is directed towards the west. At all, except low latitudes, a reversal of this latter component occurs during summer. During this season also a small southerly component is evident in temperate latitudes and a northerly component appears at high latitudes. Observed velocities are of the order 15 to $50 \mathrm{~m} / \mathrm{s}$. It is significant that observed directions of movement are not consistent with those observed by Harvey [1955] using the technique described in section 2.1 above, which apply specifically to $E_{s}$ movement. It would appear therefore that observed movements in $E$ region and $E_{s}$ movement are not compatible.

\subsection{Refraction of Radio Signals}

Perturbations in the ionosphere may also be studied by observing refraction of radio signals as they pass through the medium. Satellites or radio stars provide satisfactory discrete sources and signals from these often show short period variations in intensity known as scintillations, attributed to the presence or apparent motion of small perturbations in the plasma [Hewish, 1952; Munro, 1963; Yeh and Swenson, 1959]. There is a strong correlation between this phenomenon and that of spread $F$ observed on vertical incidence ionograms. Radio star observations at spaced sites indicate similarity in scintillation patterns, satisfactory correlation being obtained with site spacings of several kilometers. Corresponding time differences between similar phases of scintillation fading may therefore be used to estimate speed and direction of movement of perturbations.
Lateral extent of irregularities, based on the assumption that the diffracting screen is thin, is usually estimated as between 2 and $10 \mathrm{~km}$ [Hewish, 1952]. However, observations have shown that perturbations are present over a range of heights and regions of occurrence are thick [Munro, 1963; McClure, 1964]. Topside soundings [Calvert and Schmid, 1964] even show the presence of spread $F$ above the maximum ion density of the medium, hence the basic thin screen assumption does not appear valid and derived values of perturbation size are somewhat doubtful.

The phenomenon is mainly observed at night when direction of movement which refers to $F$ region is predominately towards the west. Measured speeds range usually from $70 \mathrm{~m} / \mathrm{s}$ to $120 \mathrm{~m} / \mathrm{s}$, but these have been observed to increase during periods of magnetic activity to values as high as $500 \mathrm{~m} / \mathrm{s}$. It is a feature of the phenomena that the contributing irregularities occur in well defined patches within the ionospheric medium. These can be conveniently examined by satellite observations. Munro [1963] has used this technique to show the presence of patches some hundreds of miles in extent in the north-south direction and elongated along an eastwest line. The diurnal, seasonal, latitudinal and height variations of scintillations observed on satellite transmissions have been extensively studied by Lawrence and Martin [1964].

\subsection{Backscatter Observations}

Unlike methods previously discussed which rely on vertical incidence observations of the ionosphere, this method of observing ionospheric perturbations relies on pulse signals received at oblique incidence from the medium "backscattered" from the ground at some considerable distance from the observing point. Often the range of the returned pulse echo changes rapidly with time and this has been interpreted as being due to changes in the shape of effective reflecting surfaces in the ion density plasma during the apparent passage of an ion density perturbation.

Valverde [1958] has used this technique to show the presence of very large perturbations in the $F$ region. Some of these have been shown to travel distances in excess of 4000 kilometers and to have wave fronts of the same order of magnitude. Estimated speeds which ranged from 200 to $550 \mathrm{~m} / \mathrm{s}$ are considerably higher than speeds observed for similar perturbations by Heisler [1958] discussed below. Observations, which were mainly during daylight hours in winter, showed directions consistently towards the south.

Tveten [1961] has made observations using a similar technique during daylight hours in winter, however his results refer to smaller perturbations or ripple structure in the medium with apparent separation between events of the order $180 \mathrm{~km}$. Tveten obtained median speeds of movement of the order $160 \mathrm{~m} / \mathrm{s}$, the direction of movement again being. directed towards the south. 
This technique has also been used to study apparent motion of sporadic $E$ patches. Clark and Petersen [1956] have observed these movements at Stanford $\left(37^{\circ} 26^{\prime} \mathrm{N}, 122^{\circ} 10^{\prime} \mathrm{W}\right)$. Motion was directed predominately towards the west with speeds of the order $70 \mathrm{~m} / \mathrm{s}$. Harwood [1961] has also used this method to observe $E_{s}$ movement at Slough $\left(50^{\circ} 31^{\prime} \mathrm{N}, 55^{\prime} \mathrm{W}\right)$. Patches observed had an average diameter of 200 kilometers and moved towards the southwest with speeds of the order $60 \mathrm{~m} / \mathrm{s}$. It will be noticed that directions of movement are inconsistent with those observed by Clark and Petersen and both observations do not agree with those made by Harvey in section 2.1 above. This is probably due to the different geographical locations of observing sites.

\section{Variable Frequency Observation of Ionospheric Perturbations}

A perturbation in ionospheric plasma is not confined to a specific value of ion density. It occurs over a range of ionospheric heights in a medium where there is invariably a continuous change of ion density with height. The single frequency observations discussed in section 2, therefore only provide limited information on the extent and variation of the perturbation within the medium. A complete representation of the irregularity can only be obtained therefore by studying changes over a range of ion density values such as provided by ionograms or swept frequency ionospheric records.

The perturbations are manifested on these records as complexities in the virtual height information or as apparent additional stratifications, which decrease in virtual height during successive ionograms. The perturbation therefore always has an apparent vertical component of progression.

The various forms have been classified by the author [Heisler, 1958] who has suggested that the type of anomaly observed is a function of the particular ion density distribution in which the irregularity appears. During winter months the associated anomaly appears as a readily recognizable additional stratification or "cusp" at a large vertical height above the normal $F_{2}$ stratification. This is always preceded by a fall in $F_{2}$ critical frequency and subsequently the cusp decreases in height, eventually assuming the appearance of a new $F_{2}$ stratification. The phenomenon has been explained in terms of a nonvertical reflection mechanism by Munro and Heisler [1956a]. This particular feature on ionograms has been extensively studied at this laboratory. A time difference is always observed between appearance of the cusp at the same virtual height on the ordinary and extraordinary ray traces [Munro and Heisler, 1956b], which is indicative of an apparent horizontal component of progression of the contributing perturbation through the medium. This observation incidentally provides strong evidence that perturbations are in fact occurring in the $F$ region and that observed effects are not due to ray interaction with irregularities in lower parts of the medium. Observed time differences are consistent with speeds of movement of irregularities and expected separation of reflection points in the $F_{2}$ region. As mentioned previously, a vertical component of progression is always present. This is interpreted as a tilt in the moving wave front of the disturbance.

From observations at spaced recording sites it is possible to obtain speeds and direction of movement of perturbations. Studies using ionograms from Australian stations [Heisler, 1958] have shown that the irregularities giving rise to this particular form of anomaly are a very extensive frontal phenomena traveling distances of some thousands of kilometers with little change in form. Amplitudes are such that changes in ion density of the order 30 percent are frequently observed and they possess a lateral extent in their direction of travel of the order 400 to $600 \mathrm{~km}$. Apparent motion is directed almost due north with a mean velocity of $160 \mathrm{~m} / \mathrm{s}$. Recently, observations of the anomaly on topside ionograms [Faynot and Vila, 1964] show that these large irregularities extend to considerable heights in the medium.

Wells [1957] has derived directions and speeds from observations of similar anomalies on ionograms taken near Washington, D.C., on the east coast of the United States. A considerable scatter of observed directions was obtained, however no movements were observed in the southwest sector and a predominant southeast movement prevailed. Observed velocities ranged between 60 and $150 \mathrm{~m} / \mathrm{s}$. Thomas [1959] has used spaced ionosondes in the vicinity of Slough, England, observing time differences between change of $F_{2}$ critical frequency as a means of deriving information on perturbation movement. A median speed of $155 \mathrm{Mc} / \mathrm{s}$ was found for motion directed $150^{\circ}-170^{\circ}$ east of north during winter and $120^{\circ}-140^{\circ}$ east of north during summer.

This method of observing perturbations has not been used extensively for studies in the $E$ region. Harwood [1961] from observations using a backscatter technique (sec. 2.4) has indicated that $E_{s}$ patches may not move within the medium but grow and decay in situ, probably due to some form of perturbing wave motion. This proposed mechanism and many aspects of sporadic $E$ formation could be studied to advantage by using closely spaced ionosonde observations.

Observed anomalies on ionograms change rapidly during advent of an ionospheric perturbation. It is therefore essential for a rapid sequence of records (at least one a minute) to be used in order to fully resolve the event, and to ensure accurate timing of similar phases of the event at spaced observing sites. Studies have recently been extended at this laboratory by observations made with a new ionosonde [Heisler and Wilson, 1961; Wilson, 1962]. This equipment has an electronic scan facility which provides a wide choice of scan range, scan rate, and repetition rates by easily adjusted front panel controls. Similar controls also permit selection of either a logarithmic or linear variation of frequency scan with time. 
By producing ionograms which only cover a small swept frequency range small anomalies become apparent which would not be obvious on conventional records. Such an ionogram is shown in figure 1 . This is a linear 10 sec scan covering the frequency range 7 to $9 \mathrm{Mc} / \mathrm{s}$. It is doubtful whether the spliting detail in the ordinary and extraordinary ray traces would be evident on the usual ionogram covering the full frequency range. On successive ionograms the virtual height of the complexities decrease, corresponding to the vertical component of progression usually associated with pertubation movement, while the difference in virtual height of the anomaly on the ordinary and extraordinary ray traces is evidence of the associated horizontal component of progression. The contributing perturbation is much smaller than that observed on conventional ionograms and probably corresponds to those observed by Munro (sec. 2.1 above).

An example of the rapid scan facility of the recorder is shown in figure 2. These are two 2-sec linear scan ionograms taken from a series of records produced at a repetition rate of 20 a minute. It will be noticed that $f_{\min }$ which has a value less than $1.5 \mathrm{Mc} / \mathrm{s}$ on the first ionogram has increased to $1.9 \mathrm{Mc} / \mathrm{s}$ in the second ionogram. The two ionograms are five frames apart, therefore a change of $0.4 \mathrm{Mc} / \mathrm{s}$ at least has occurred in the value of $f_{\mathrm{min}}$ in $15 \mathrm{sec}$. Unfortunately, because of localized noise and interference due to broadcast stations at the present site, it is only possible to record these changes when the average value of $f_{\min }$ is high. However there are indications that rapid changes in $f_{\min }$ occur quite frequently. The phenomenon, which is being investigated further, may be due to focusing caused by perturbations in the $E$ region, or may be a genuine absorption effect related to the presence of irregularities in the $D$ region, for instance Gardner and Pawsey [1953] report curious disturbances operative for a few seconds which cause extended regions in the lower ionosphere at $70 \mathrm{~km}$ to produce echoes.

Similar records to those shown in figure 2 have been used to study rapid variations in sporadic $E$ ionization. Changes in $E_{s}$ critical frequency of the order of a megacycle have been observed over a period of several minutes, moreover in successive ionograms this is accompanied by a breaking up of the $E_{s}$ trace at a frequency about $0.5 \mathrm{Mc} / \mathrm{s}$ below $f_{0} E_{s}$. The phenomenon has been fully described by Heisler and Whitehead [1962] and is attributed to Fresnel diffraction effects from patches of ionization with apparent movement within the medium.

A recent modification to the equipment has enabled similar ionograms to be recorded with amplitude deflection in the direction of the height axes. This has enabled a study to be made of rapid variations in amplitude of received pulse signals over a range of frequencies [Heisler, 1964]. The usual form of $F$ region perturbations already discussed were present, manifested as bursts of amplitude due to focusing processes, proceeding downwards through the observed frequency range. However, the presence of small perturbations was also disclosed pro- gressing rapidly upwards through the medium. Investigations are proceeding to see whether those irregularities observed by the Mitra method, as explained in section 2.2 above, belong to the latter category. Evidence was also forthcoming to suggest that horizontal extent of an $F$ region perturbation increases as it progresses into the lower ion density regions of the medium.

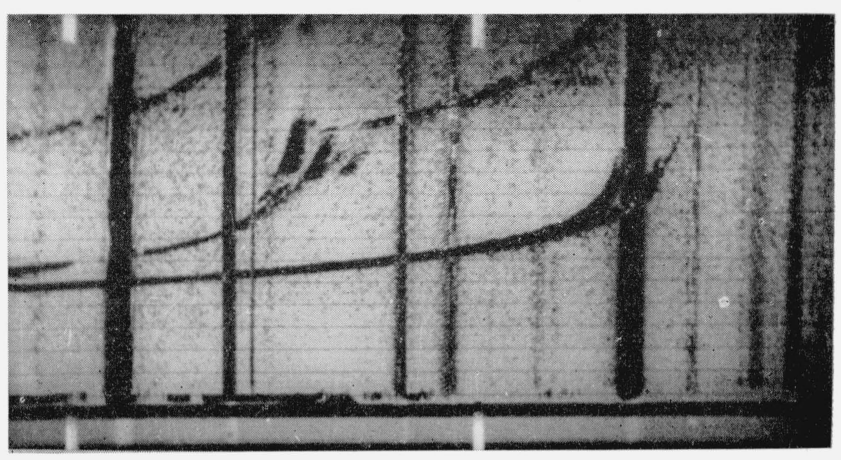

Figure 1. A linear ten second scan ionogram covering the frequency range 7 to $9 \mathrm{Mc} / \mathrm{s}$.
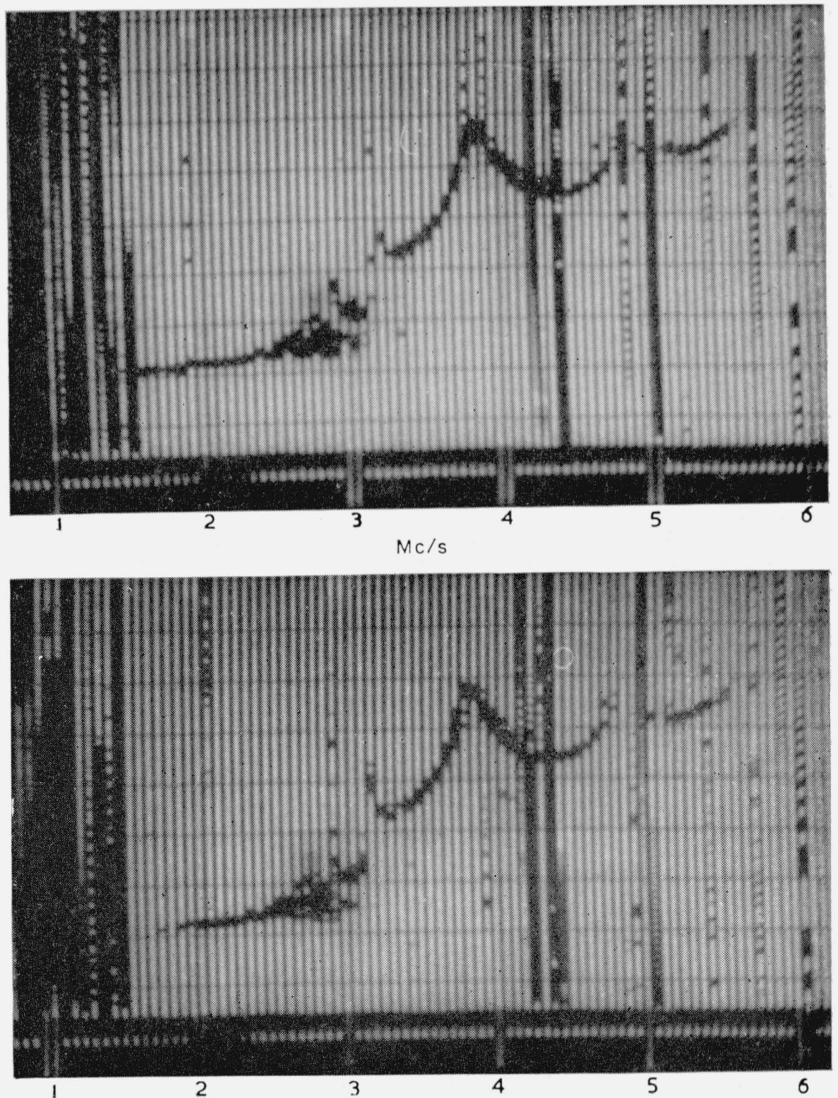

Figure 2. Two second linear scan ionograms from a series taken at a repetition rate of twenty scans a minute showing rapid variations in $f_{\mathrm{min}}$. 


\section{Discussion}

The primary purpose of experimental investigations is to provide facts and data to assist the theorist in developing a hypothesis to explain the observed phenomena. Despite the information which has been gathered over a period of years on perturbations in ionospheric plasma, this is still insufficient to enable satisfactory interpretation to be made of observed effects. It must be stressed that observed movement is apparent only and reference to this as a drift or ionospheric wind is assumptive. In the $F$ region winds have negligible effect on east-west ionization movement and only affects north-south horizontal movement over comparatively large distances at low latitudes. Appreciable drift is only possible in an east-west direction and then only by the influence of an applied north-south electrostatic field [Martyn, 1953]. Martyn [1954] has postulated the existence of such a field communicated from the mean conducting path in the $E$ region along lines of magnetic force, and there is considerable evidence to suggest that this does account for observed east-west motion in $F$ region [Heisler, 1960]. However, it is hard to account for large observed movement in a north-south direction at all latitudes unless this is due to some form of perturbing waveform traveling through the medium. Observed seasonal and diurnal variations in speeds and direction of movement in $F$ region can be conveniently explained on this basis [Heisler, 1963.]

These restrictions do not apply to the lower $E$ region where ionization can move horizontally with substantial local wind velocity [Martyn, 1953]. Visual observations of meteor trails [Millman, 1959; Greenhow and Neufeld, 1959] have firmly established the existence of winds between 80 and 100 $\mathrm{km}$, and it seems reasonable to assume that these would also appear in the 100 to $120 \mathrm{~km}$ height range. It is conceivable therefore that observed movement in $E$ region as measured by the Mitra method could in fact refer to a true wind within the medium, perturbations being introduced by some form of turbulance mechanism. There is some doubt however as to whether observed motion of $E_{s}$ patches is due to a material drift or wind. Harwood [1961] for instance using backscatter observations has found no consistent correlation between apparent movement of patches and movement derived from simultaneous Mitra method measurements; furthermore, he has observed simultaneously occurring $E_{s}$ clouds a few hundreds of kilometers apart drifting in quite different directions. The reported observation by Heisler and Whitehead [1960] and Bowman [1960] of a correlation between large $F$ region perturbations and occurrence of sporadic $E$ would also suggest some form of motion not necessarily related to winds or drifts.

One significant feature which is apparent in results discussed in this paper is the marked discrepancy between speeds and directions of perturbations measured by different observational techniques. This is most obvious for instance in $F$ region param- eters obtained by the Mitra technique, and has been explained as a function of data selectivity controlled by the particular observational method [Heisler, 1963]. Furthermore there is much evidence to suggest that many manifestations of the phenomenon are frontal in form. It is usually assumed that direction of movement is perpendicular to the observed front, however there is no evidence to indicate that this need necessarily be so. Certainly in the case of very large frontal phenomena, any movement in a direction parallel to the front would be effectively masked except where irregularities in frontal shape occurred which were comparable in size with spacing of observing sites. It would appear that much knowledge of perturbations within the plasma could be obtained by use of different techniques conjointly at a number of well chosen geographical locations. At all times careful consideration must be given to physical significance of observations and particularly to comparisons made between data derived by different experimental methods.

This work has been carried out in the School of Electrical Engineering of the University of Sydney as part of the research program sponsored by the Radio Research Board of the Commonwealth Scientific and Industrial Research Organization. Appreciation is expressed to the University of Sydney and in particular to Professor W. N. Christiansen for provision of facilities in the School of Electrical Engineering. The author also thanks Dr. G. H. Munro for helpful discussions during the preparation of the paper.

\section{References}

Bowman, G. G. (1960), Some aspects of sporadic E at mid latitudes, Planetary Space Sci. 2, 195-211.

Briggs, B. H. (1960), A preliminary report on horizontal drifts in the F region during IGY, p. 297. Some Ionospheric Results-IGY (Elsevier Publishing Company, New York, N.Y.).

Briggs, B. H., and M. Spencer (1954), Horizontal movements in the ionosphere, Rept. Progr. Phys. 17, 245-280.

Calvert, W., and C. W. Schmid (1964), Spread-F observations by the Alouette topside sounder satellite, J. Geophys. Res. 69, No. 9, 1839-1852.

Clark, C., and A. H. Peterson (1956), Motion of sporadic E patches determined from high-frequency backscatter records, Nature Lond. 178, No. 4531, 486.

Faynot, J. M., and P. Vila (1964), Sur certaines perturbations itinerantes observees dans l'ionosphere inferieure et superieure, Centre National d'estudes des Telecommunications, Group de Recherches Ionospheriques Note Technique G RI/NT/20.

Gardner, F. F., and J. L. Pawsey (1953), Study of the ionosspheric D-region using partial reflections, J. Atmospheric Terrest. Phys. 3, 321-344.

Greenhow, J. S., and E. L. Neufield (1959), Measurements of turbulence in the 80 to $100 \mathrm{~km}$ region from the radio echo observation of meteors, J. Geophys. Res. 64, No. 12, 2129-2133.

Harvey, J. H. (1955), Movement of sporadic E ionization, Aust. J. Phys. 8, No. 4, 523-534.

Harwood, J. (1961), Some observations of the occurrence and movement of sporadic E ionization, J. Atmospheric Terrest. Phys. 20, No. 4, 243-261. 
Heisler, L. H. (1958), Anamolies in ionosonde records due to travelling ionospheric disturbances, Aust. J. Phys. 11, No. 1, 79-90.

Heisler, L. H. (1960), A relation between ionospheric drifts and atmospheric dynamo current systems, Aust. J. Phys. 13, No. 2, 188-191.

Heisler, L. H. (1963), Observation of movement of perturbations in the $\mathrm{F}$ region, J. Atmospheric Terrest. Phys. 25, $71-86$

Heisler, L. H. (1964), The observation of amplitude variations in swept frequency signals from the ionospheric $\mathrm{E}$ and $\mathrm{F}$ regions, J. Atmospheric Terrest. Phys., in press.

Heisler, L. H., and J. D. Whitehead (1960), F region travelling disturbances and sporadic E ionization, J. Geophys. Res. 65, No. 9, 2767-2773.

Heisler, L. H., and J. D. Whitehead (1962), Rapid variations in the sporadic E region, J. Atmospheric Terrest. Phys. 24, $753-764$.

Heisler, L. H., and L. D. Wilson (1961), Design of panoramic ionospheric recorders, J. Res. NBS 65D (Radio Prop.), No. 6, 629-636.

Hewish, A. (1952), The diffraction of galactic radio waves as a method of investigating the irregular structure of the ionosphere, Proc. Roy. Soc. (London) Ser. A, 214, 494-514.

Lawrence, J. D. Jr., and J. D. Martin (1964), Diurnal, seasonal, latitudinal, and height variations of satellite scintillations, J. Geophys. Res. 69, No. 7, 1293-1300.

Martyn, D. F. (1953), Ionization drift due to winds and electric fields, Phil. Trans. Soc. (London), Ser. A 246, 306320.

Martyn, D. F. (1954), Interpretation of observed $\mathrm{F}_{2}$ 'winds' as ionization drifts associated with the magnetic variations, Rept. of the Physical Soc. Conference on the Physics of the Ionosphere, 163-165 (Physical Society, London).

McClure, J. P. (1964), The height of ionospheric irregularities in temperate latitudes, J. Geophys. Res. 69, No. 13, $2775-2780$.

Millman, P. M. (1959), Visual and photographic observations of meteors and noctilucent clouds, J. Geophys. Res. 64,21222128.

Mitra, S. N. (1949), A radio method of measuring winds in the ionosphere, Proc. IEE, Pt. III, 96, 441-446.

Munro, G. H. (1950), Travelling disturbances in the ionosphere, Proc. Roy. Soc. (London) Ser. A 202, 208-223.

Munro, G. H. (1953), Reflections from irregularities in the ionosphere, Proc. Roy. Soc. (London), Ser. A 219, 447-463.

Munro, G. H. (1958), Travelling ionospheric disturbances in the F region, Aust. J. Phys. 11, 91-112.

Munro, G. H. (1963), Scintillation of radio signals from satellites, J. Geophys. Res. 68, 1851-1860.
Munro, G. H., and L. H. Heisler (1956a), Cusp type anomalies in variable frequency ionospheric records, Aust. J. Phys. 9, No. 3, 343-358.

Munro, G. H., and L. H. Heisler (1956b), Divergence of radio rays in the ionosphere, Aust. J. Phys. 9, No. 3, 359-372.

Phillips, G. J. (1955), An apparatus for recording time-delays between radio fading characteristics, J. Atmospheric Terrest. Phys. 6, 124-128.

Price, R. E. (1955), Traveling disturbances in the ionosphere, Rept. of the Physical Soc. Conference on the Physics of the Ionosphere, 181-190 (Physical Society, London).

Ratcliffe, J. A. (1956), Some aspects of diffraction theory and their application to the ionosphere, Rept. Progr. Phys. 19, $188-267$

Shimizaki, T. (1959), World wide measurements of horizontal ionospheric drifts, Rept. Ionos. Space Res. (Japan) 13, $21-47$.

Shimizaki, T. (1960), A comparison of horizontal ionospheric drifts at different latitudes, some ionospheric results obtained during the IGY, 345-354 (Elsvier Publishing Co., New York, N.Y.).

Thomas, L. (1959), Some measurements of horizontal movements in region- $\mathrm{F}_{2}$ using widely spaced observing stations, J. Atmospheric Terrest. Phys. 14, 123-137.

Titheridge, J. E. (1963), Large scale irregularities in the ionosphere, J. Geophys. Res. 68, 3399-3417.

Toman, K. (1955), Movement of the F region, J. Geophys. Res. 60, $57-70$.

Tveten, L. H. (1961), Ionospheric motions observed with high frequency backscatter sounders, J. Res. NBS 65D (Radio Prop.), No. 2, 115-127.

Valverde, J. F. (1958), Motions of large scale traveling disturbances determined from high-frequency backscatter and vertical incidence records. Radio Propagation Laboratory, Stanford University Scientific Rept. No. 1.

Wells, H. W. (1957), Large scale movements of the layers, J. Atmospheric Terrest. Phys. Special supplement, 33-40.

Wilson, L. D. (1962), An electronically scanned panoramic ionospheric recorder, Aust. J. Appl. Science 13, No. 2, 89-97.

Yeh, K. C., and G. W. Swenson, Jr. (1959), The scintillation of radio signals from satellites, J. Geophys. Res. 64, No. 12, $2281-2286$.

(Paper 69D2-456) 